\title{
Hidradenitis suppurativa - known and unknown disease
}

\section{Marta Wieczorek, Irena Walecka}

Department of Dermatology, Medical Centre for Postgraduate Education, Central Hospital
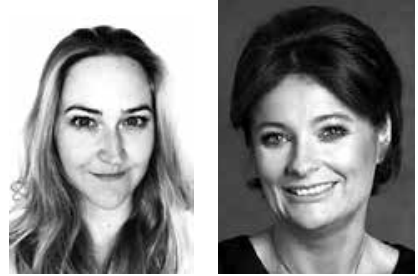

of Ministry of Internal Affairs, Warsaw, Poland

Hidradenitis suppurativa (HS) is a chronic and recurrent inflammatory skin disease that is also known as acne inversa and, historically, as Verneuil's disease. The prevalence of $\mathrm{HS}$ varies, ranging from less than $1 \%$ to $4 \%$ [1].

The onset of disease symptoms mostly takes place between puberty and age 40, peaking in the second or third decade of life. Women are more likely to develop HS than men. A study in the United States found that the frequency of HS was twice as high among women than among men; the highest age-specific incidence was among patients between the ages of 18 and 29 years [2]. The higher incidence of HS was also observed in patients with spondyloarthritis and the onset of HS symptoms was associated with increased SpA activity [3, 4].

The cause of hidradenitis suppurativa is unclear and multifactorial. Various factors have an impact on the follicular epithelium, including genetic susceptibility, obesity, diet, mechanical stresses on the skin, smoking, and hormonal factors. Genetics plays a role in up to $40 \%$ of patients with HS (positive family history) [5]. As in acne vulgaris, hormonal changes connected with obesity may result in androgen excess and increase follicular plugging. There is a very strong relationship between smoking and hidradenitis suppurativa - most affected patients are active smokers. Hidradenitis suppurativa was traditionally viewed as a disease of the sweat glands, but the latest research suggests that it is a disorder of follicular epithelium connected with repeated mechanical stress in genetically liable individuals. The first progression for the development of HS lesions is connected with follicular occlusion. Follicular occlusion appears to result from ductal keratinocyte proliferation (follicular epithelial hyperplasia), causing follicular hyperkeratosis and plugging [6].

Hidradenitis suppurativa typically presents with inflammatory nodules, abscesses, comedones, sinus tracts and scarring. HS can affect the axillae (most commonly), inguinal area, inner thighs, perianal areas, mammary and inframammary regions, buttocks, pubic region, scrotum, vulva, trunk, and, occasionally, the scalp and retroauricular areas [7]. Early beginning of skin changes may be associated with a greater risk for extensive disease. Sex influences the distribution of HS. In women primary changes develop in the groin or upper inner thigh, axilla, chest (including breast and inframammary regions), and the buttocks or gluteal clefts. Meanwhile in men, primary sites include the groin or thigh, axilla, perineal or perianal regions, and buttocks or gluteal cleft. Other common locations are beltlines, waistbands, abdominal folds, and brassiere straps or bands. Prodromal symptoms may also be experienced prior to flares, such as fatigue and localized pruritus or paresthesias. The primary visible lesions of HS are inflammatory nodules. Sinus formation, clusters of open comedones (tombstone comedones), and scarring are the result of recurrent or persistent disease.

The Hurley clinical staging system is used to classify patients with HS into three disease severity groups.

- Stage I- abscess formation (single or multiple), no sinus tracts or cicatrization/scarring.

- Stage II - recurrent abscesses with sinus tracts and scarring, single or multiple separated lesions.

- Stage III - diffuse or almost diffuse involvement, or multiple interconnected sinus tracts and abscesses across the entire area [8] (Fig. 1).

Hidradenitis suppurativa has a major impact on patient quality of life. Pain and scarring are a significant cause of disutility, cause the necessity of dressings, and have an accompanying odor, which are embarrassing and humiliating. As a result, patients experience social isolation, depression, and loss of employment. Also increased suicide risk has been reported [9].

A diagnosis of HS is immediate in patients who demonstrate the patient history and recognition of characteristic clinical manifestations: 

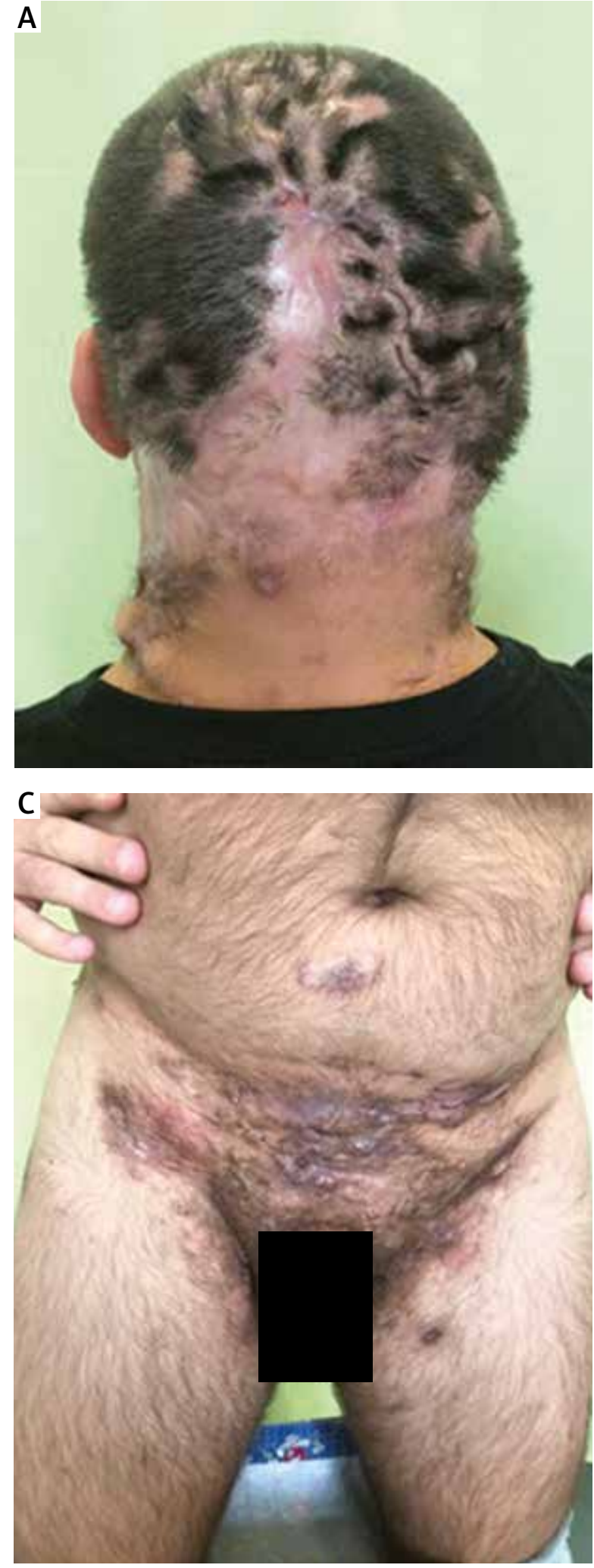

Fig. 1. Hidradenitis suppurativa - Hurley stage III.

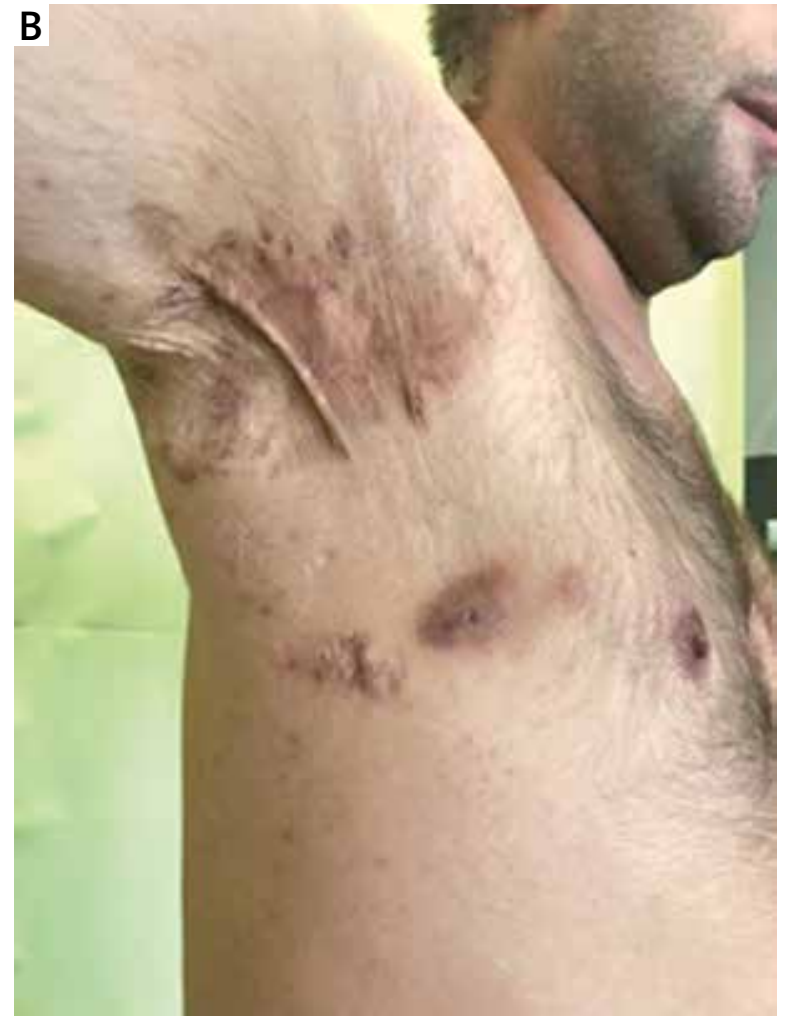

- Typical lesions (multiple inflamed nodules, tombstone comedones, sinus tracts, abscesses and/or fibrotic scars). A full skin examination should be performed in patients with suspected HS.

- Typical locations (in particular, axillae, groin, inframammary areas; often bilateral distribution).

- The patient history is a valuable tool in the diagnosis of HS (onset in adolescence or young adulthood, a history of recurrent or persistent disease, or a family history).

This may allow for diagnosis of the disease at early stages.

A skin biopsy usually is not necessary for diagnosis. In cases in which the diagnosis is indefinite, a biopsy can be useful for excluding other disorders. Routine bacterial cultures are not advisable. Ultrasound imaging may be useful for some cases [10].

Patient education (lifestyle modifications) and support are essential components of treatment [11]. The clinician should evaluate the patient's quality of life and estimate symptoms of depression at each visit.

Patient self-management is an important part of treatment:

- Avoiding unnecessary skin trauma and irritation: factors that promote skin maceration and trauma may add to worsening of HS. 
- Smoking discontinuation.

- Weight loss.

- Topical antiseptic washes.

Hidradenitis suppurativa is difficult to treat and there is no cure for this condition, but there are effective treatments available to control the disease and improve symptoms. Goals of HS treatment include preventing new lesions, treating newly formed lesions and removing existing nodules and sinus tracts. Treatment interventions are based on the disease severity according to the Hurley staging.

\section{Hurley stage I - mild disease}

Local therapy combined with topical clindamycin, intralesional corticosteroid injections, topical resorcinol and punch debridement. When insufficient, oral tetracyclines may be beneficial.

\section{Hurley stage II - antibiotic therapy}

Oral tetracyclines (500 mg twice daily) are a key treatment for mild to moderate HS especially when there are no inflammatory abscesses for up to 4 months.

Combination therapy with clindamycin (300 mg twice daily) and rifampin (600 mg once daily) for 10 weeks is an option for patients who fail to respond to oral tetracyclines.

Other antibiotics, oral retinoids and hormonal therapy often do not bring satisfactory results.

\section{Hurley stage III - severe and refractory disease}

Patients who do not respond to the above therapies may benefit from biologic treatments (adalimumab or infliximab).

Surgery can be used for the treatment of individual nodules and sinus tracts that occur in any Hurley stage of disease. Wide excision is typically used for Hurley stage III disease [12]

The authors declare no conflict of interest.

\section{References}

1. Alikhan A, Lynch PJ, Eisen DB. Hidradenitis suppurativa: a comprehensive review. J Am Acad Dermatol 2009; 60: 539-561.

2. Garg A, Lavian J, Lin G, et al. Incidence of hidradenitis suppurativa in the United States: A sex- and age-adjusted population analysis. J Am Acad Dermatol 2017; 77: 118-122.

3. Richette P, Molto A, Viguier M, et al. Hidradenitis Suppurativa Associated with Spondyloarthritis - Results from a Multicenter National Prospective Study. J Rheumatol 2014; 41: 490-494.

4. Rondags A, Arends S, Wink FR, et al. High prevalence of hidradenitis suppurativa symptoms in axial spondyloarthritis patients: A possible new extra-articular manifestation. Semin Arthritis Rheum 2018; pii: S0049-0172(18)30015-5.

5. Pink AE, Simpson MA, Desai N, et al. Mutations in the $\gamma$-secretase genes NCSTN, PSENEN, and PSEN1 underlie rare forms of hidradenitis suppurativa (acne inversa). J Invest Dermatol 2012; 132: 2459-2461.

6. von Laffert M, Stadie V, Wohlrab J, Marsch WC. Hidradenitis suppurativa/acne inversa: bilocated epithelial hyperplasia with very different sequelae. Br J Dermatol 2011; 164: 367-371.

7. Slade DE, Powell BW, Mortimer PS. Hidradenitis suppurativa: pathogenesis and management. Br J Plast Surg 2003; 56: 451-461.

8. Vazquez BG, Alikhan A, Weaver AL, et al. Incidence of hidradenitis suppurativa and associated factors: a population-based study of Olmsted County, Minnesota. J Invest Dermatol 2013; 133: 97-103.

9. Onderdijk AJ, van der Zee HH, Esmann S, et al. Depression in patients with hidradenitis suppurativa. J Eur Acad Dermatol Venereol 2013; 27: 473-478.

10. Jemec GB. Clinical practice. Hidradenitis suppurativa. N Engl J Med 2012; 366: 158-164

11. Sartorius K, Emtestam L, Jemec GB, Lapins J. Objective scoring of hidradenitis suppurativa reflecting the role of tobacco smoking and obesity. Br J Dermatol 2009; 161: 831-839.

12. Gulliver W, Zouboulis CC, Prens E, et al. Evidence-based approach to the treatment of hidradenitis suppurativa/acne inversa, based on the European guidelines for hidradenitis suppurativa. Rev Endocr Metab Disord 2016; 17: 343-351. 